DOI 10. 18307/2018. 0224

(c) 2018 by Journal of Lake Sciences

\title{
基于面平均雨量误差修正的实时洪水预报修正方法
}

\author{
司 伟 ${ }^{1,2}$,包为民 ${ }^{2}$,㫿思敏 ${ }^{2}$, 石 朋 $^{2}$ \\ (1:河海大学商学院,南京 210098) \\ $(2$ :河海大学水文及水资源学院,南京 210098$)$
}

\begin{abstract}
摘 要: 空间集总式水文模型的洪水预报精度会受到面平均雨量估计误差的严重影响. 点雨量监测值的误差类型、误差 大小以及流域的雨量站点密度和站点的空间分布都会影响到面平均雨量的计算. 为提高实时洪水预报精度, 本文提出了 一种基于降雨系统响应曲线洪水预报误差修正方法. 通过此方法估计降雨输人项的误差, 从而提高洪水预报精度. 此方 法将水文模型做为输人和输出之间的响应系统, 用实测流量和计算流量之间的差值做为信息,通过降雨系统响应曲线, 使用最小二乘估计原理,对面平均雨量进行修正,再用修正后的面平均雨量重新计算出流过程. 将此修正方法结合新安 江模型使用理想案例进行检验,并应用于王家坝流域的 16 场历史洪水以及此流域不同雨量站密度的情况下,结果证明均 有明显修正效果, 且在雨量站密度较低时修正效果更加明显. 该方法是一种结构简单且不增加模型参数和复杂度的实时 洪水修正的新方法.
\end{abstract}

关键词: 实时修正;面平均雨量误差;降雨系统响应曲线;新安江模型;王家坝流域

\section{Real-time flood forecast updating method based on mean areal rainfall error correction}

\author{
SI Wei ${ }^{1,2}$, BAO Weimin ${ }^{2}$, QU Simin ${ }^{2} \&$ SHI Peng ${ }^{2}$ \\ (1: Business School of Hohai University, Nanjing 210098, P.R. China) \\ (2: College of Hydrology and Water Resources, Hohai University, Nanjing 210098, P.R.China)
}

\begin{abstract}
The accuracy of flood forecasts generated using spatially lumped hydrological models can be severely affected by errors in the estimates of mean areal rainfall. The quality of the latter depends both on the size and type of errors in point-based rainfall measurements, and on the density and spatial arrangement of rain gauges in the basin. Here, we use error feedback correction, based on the rainfall system response curve method, to compute updated estimates of the rainfall inputs. The capability of the method to improve the accuracy of real-time flood forecasts is demonstrated using the Xin'anjiang (XAJ) model applied to 16 flood events of the Wangjiaba Basin. The result shows that the forecast improvement is significant. For the Wangjiaba Basin, we also examinethe performance of the method for different rain gauge densities, and find that forecast improvement is more significant when gauge densities are lower. The method is relatively simple to apply and can improve the accuracy of real-time model predictions without increasing either model complexity and/or the number of model parameters.
\end{abstract}

Keywords: Real-time updating; mean areal rainfall error; system response curve; Xin'anjiang model; Wangjiaba Basin

由于流域降雨径流过程受到降雨和下垫面条件等多重因素影响, 实时洪水预报精度难以满足要求. 预 报模型初始状态值误差 ${ }^{[1]}$ 、模型输人误差 ${ }^{[2]}$ 、模型结构的不充分性 ${ }^{[3]}$ 以及参数非优化 ${ }^{[4]}$ 都会影响预报结果 的精度. 所以经常使用误差修正技术来减少模型在预报时产生的误差 ${ }^{[5-6]}$.

降雨径流过程涉及蒸发、下渗、产流和汇流等多个环节,影响因素复杂. 简单的误差修正方法是通过流 量误差直接对模型计算结果 (流量过程) 进行修正 ${ }^{[7]}$. 组合卡尔曼滤波 (EnKF) 或者粒子滤波 $(P F)$ 方法经常 通过数据同化技术对模型初始值或者模型参数进行修正,然而研究结果表明滤波方法在预报修正中的效果 过于自信, 对于模型预报的不确定性估计不足 ${ }^{[8-9]}$. 有学者尝试使用滤波方法对降雨资料误差进行估计和修

* 国家自然科学基金项目 (51709077) 和博士后基金项目 (2017M611679/1701019A) 联合资助. 2017-03-31 收稿; 2017-06-26 收修改稿. 司伟(1987 ), 男,博士; E-mail: lindongsisi@ 163.com. 
正,但结果显示仅有在土壤含水量很低且流量资料非常精确的情况下才有明显效果 ${ }^{[10]}$.

影响实时洪水预报系统的误差因素较多, 其影响机理非常复杂 ${ }^{[11]}$. 产流量是衡量洪水预报水量平衡的 重要指标,笔者曾通过直接修正产流量来提高洪水预报精度 ${ }^{[12-14]}$. 但引起产流量计算误差的因素较多,通过 对这些因素的归纳分析可以发现, 面平均雨量是洪水预报模型的最重要输人项, 面平均雨量误差直接影响 初始值和计算产流量, 从而影响模型预报精度 ${ }^{[15]}$, 对于集总式水文模型尤为明显. 降雨过程是受到一系列 因素 (气候、地形、植被以及人类活动) 影响的随机过程, 此类误差的特性以及误差的大小难以预测和估 计 ${ }^{[16]}$. 由于遥感降雨资料本身具有误差, 卫星和雷达降雨资料的使用同样无法解决降雨资料误差的问 题 ${ }^{[17]}$. 集总式水文模型虽然在体现和反映真实暴雨过程剧烈的时间和空间变异性方面不如分布式水文模 型, 但由于在实际洪水预报作业过程中流域水文气象资料 (蒸发、降雨) 和流域下垫面条件 (土壤植被类型、 土壤含水量) 等无法满足分布式水文模型的要求, 所以集总式或半分布式水文模型在实时洪水预报中的应 用更广泛 ${ }^{[18]}$, 因此本研究中采用的水文模型为集总式水文模型.

因此, 本文提出一种相对简单的降雨系统响应曲线修正方法, 通过修正面平均雨量来提高模型预报精 度. 将此方法与新安江模型结合, 通过理想案例验证和实际流域应用, 并进一步检验此方法在不同雨量站密 度情况下的应用效果.

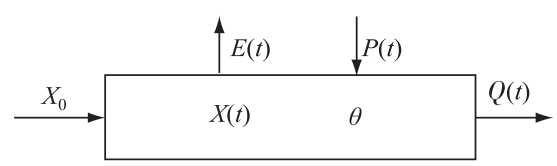

图 1 一般水文模型系统示意

Fig.1 A systems diagram of general hydrological model

\section{1 修正方法}

\section{1 降雨系统响应曲线}

将水文模型作为一般系统考虑, 图 1 中 $P(t)$ 为面平均降 雨系列, $E(t)$ 为蒸散发, $Q(t)$ 为计算的流量过程, $\theta$ 为模型参 数, $X(t)$ 是模型状态变量, $X_{0}$ 为状态初始值. 图 1 中的水文模 型系统可以表达为由输人、输出、参数和状态变量组成的系统 方程:

$$
Q(t)=f[P(t), E(t), X(t), \theta]
$$

式中, $Q=\left[q_{1}, q_{2}, q_{3}, \cdots, q_{n}\right]^{T}$ 为流域出口断面流量过程, $P=\left[p_{1}, p_{2}, p_{3}, \cdots, p_{m}\right]^{T}$ 为面平均雨量向量, $E=$ $\left[e_{1}, e_{2}, e_{3}, \cdots, e_{n}\right]^{T}$ 为蒸发, $X=\left[x_{1}, x_{2}, x_{3}, \cdots, x_{n}\right]^{T}$ 为状态变量向量, $\theta$ 为参数向量, $n$ 为时段流量系列个数, $m$ 为时段降雨系列个数.

概念性模型的绝大多数参数都具有明确的物理意义, 是流域水文特征的反应, 模型参数一旦率定之后 一般在短期之内不会改变 ${ }^{[19]}$. 模型状态变量取决于模型的输入输出项 ${ }^{[20]}$, 因此, 在本研究中认为模型参数 不变化,面平均雨量的变化是引起流域出口断面流量变化的主要因素. 上述系统方程可以简化为:

$$
Q(P)=f(P)
$$

对于流域水文模型, 把降雨输人作为自变量求全微分, 可得到式 (2) 的微分关系:

$$
\mathrm{d} Q=\left.\frac{\partial Q(P)}{\partial P}\right|_{P=P_{0}} \mathrm{~d} P
$$

式中, $\mathrm{d} P$ 为面平均雨量的变化量, $\mathrm{d} Q$ 为由面平均雨量变化量引起的流量变化量, $P_{0}$ 为修正之前的面平均雨 量系列, $\left.\frac{\partial Q}{\partial P}\right|_{P=P_{0}}$ 为系统在面平均系列为 $P_{0}$ 时的系统响应. 则观测流量可以表达为:

$$
Q(P) \approx Q\left(P_{0}\right)+\left.\frac{\partial Q(P)}{\partial p_{1}}\right|_{P=P_{0}} \Delta p_{1}+\left.\frac{\partial Q(P)}{\partial p_{2}}\right|_{P=P_{0}} \Delta p_{2}+L+\left.\frac{\partial Q(P)}{\partial p_{m}}\right|_{P=P_{0}} \Delta p_{m}
$$

式中, $Q\left(P_{0}\right)$ 为由降雨系列 $P_{0}$ 计算得到的流量系列, $\left.\frac{\partial Q(P)}{\partial p_{m}}\right|_{P=P_{0}} \Delta p_{m}$ 表示第 $m$ 时段的降雨变化量 $\Delta p_{m}$ 对出 口流量过程 $Q(P)$ 所造成的系统响应. 假设使用观测流量系列长度为 $L, Q(t)=\left[Q_{1}, Q_{2}, Q_{3}, \cdots, Q_{L}\right]^{T}$. 代人 式(4) 可得: 


$$
\left\{\begin{array}{l}
Q_{1}(P) \approx Q_{1}\left(P_{0}\right)+\left.\frac{\partial Q_{1}(P)}{\partial p_{1}}\right|_{P=P_{0}} \Delta p_{1}+\left.\frac{\partial Q_{1}(P)}{\partial p_{2}}\right|_{P=P_{0}} \Delta p_{2}+L+\left.\frac{\partial Q_{1}(P)}{\partial p_{m}}\right|_{P=P_{0}} \Delta p_{m} \\
Q_{2}(P) \approx Q_{2}\left(P_{0}\right)+\left.\frac{\partial Q_{2}(P)}{\partial p_{1}}\right|_{P=P_{0}} \Delta p_{1}+\left.\frac{\partial Q_{2}(P)}{\partial p_{2}}\right|_{P=P_{0}} \Delta p_{2}+L+\left.\frac{\partial Q_{2}(P)}{\partial p_{m}}\right|_{P=P_{0}} \Delta p_{m} \\
Q_{L}(P) \approx Q_{L}\left(P_{0}\right)+\left.\frac{\partial Q_{L}(P)}{\partial p_{1}}\right|_{P=P_{0}} \Delta p_{1}+\left.\frac{\partial Q_{L}(P)}{\partial p_{2}}\right|_{P=P_{0}} \Delta p_{2}+L+\left.\frac{\partial Q_{L}(P)}{\partial p_{m}}\right|_{P=P_{0}} \Delta p_{m}
\end{array}\right.
$$

则式 (5) 的矩阵形式为:

$$
Q(P)=Q\left(P_{0}\right)+S \cdot \Delta P+W
$$

式中, $\Delta P=\left[\Delta p_{1}, \Delta p_{2}, \Delta p_{3}, L, \Delta p_{m}\right]^{T}$ 即为面平均雨量误差的估计值; $W=\left[w_{1}, w_{2}, w_{3}, \cdots, w_{L}\right]^{T}$ 为流量观测随 机误差项,一般为服从零均值分布的为白噪声向量. $S$ 为系统响应矩阵, 其表达式为:

$$
S=\left[\begin{array}{ccc}
\frac{\partial Q_{1}(P)}{\partial p_{1}} & \cdots & \frac{\partial Q_{1}(P)}{\partial p_{m}} \\
\frac{\partial Q_{2}(P)}{\partial p_{1}} & \cdots & \frac{\partial Q_{2}(P)}{\partial p_{m}} \\
\frac{\partial Q_{L}(P)}{\partial p_{1}} & \cdots & \frac{\partial Q_{L}(P)}{\partial p_{m}}
\end{array}\right]
$$

式(7)代人式 (3)得到由降雨变化量与流量变化量表达的系统响应关系:

$$
\mathrm{d} Q=S \cdot \mathrm{d} P
$$

式(8)中 $S$ 矩阵的每一项都可以用式 $(9)$ 差分近似求解.

$$
\frac{\partial Q_{j}(P)}{\partial P_{i}}=\frac{Q_{j}\left(p_{1}, \cdots, p_{i}+\Delta p_{i}, \cdots, p_{m}\right)-Q_{j}\left(p_{1}, \cdots, p_{i}, \cdots, p_{m}\right)}{\Delta p_{i}}(i=1, \cdots, m, j=1 、 \cdots, L)
$$

式中, $Q\left(p_{1}, \cdots, p_{i}, \cdots, p_{m}\right)$ 为由修正前的面平均雨量系列计算得到的流量系列, $Q\left(p_{1}, \cdots, p_{i}+\Delta p_{i}, \cdots, p_{m}\right)$ 为由面平均雨量系列增加 $\Delta p_{i}$ 之后计算得到的流量系列.

式 (8) 中当 $i$ 不变, 而 $j$ 从 $1 \sim L$ 变化时, 该 $L$ 项差分值就是 $S$ 矩阵中的一列. 而此列正是雨量 $p_{i}$ 的单位变 化量所对应的系统响应系列, 本研究中称之为雨量 $p_{i}$ 所对应的系统响应曲线. 在实际求解系统响应曲线的 过程中, 为了计算简单, 令式 $(9)$ 中 $\Delta p_{i}=1$, 则降雨系统响应曲线就是降雨量的单位变化量引起的系统响应.

计算系统响应曲线的详细步骤如下: (1) 面平均雨量系 列 $P$ 中的时段雨量 $p_{i}(i=1 \sim m)$, 在其余时段降雨量 $p_{k}(k \neq i)$ 均不变的基础上增加 1 个单位的降雨量 $\Delta p_{i}$, 得到新的降雨系 列用 $P^{i}$ 表示. (2) 用新的面平均雨量系列 $P^{i}$ 通过模型计算后 得到流量过程 $Q_{i}(t)$. (3) 用 $Q_{i}(t)$ 减去用修正前的面平均雨 量系列 $P_{0}$ 计算得到的流量过程 $Q(t)$ 所得到的系列值, 即为 降雨量 $p_{i}$ 的系统响应曲线, 表示为 $S^{i}(t) . S$ 矩阵中的每一列 均用同样方法求得, 示意图如图 2 所示.

\section{2 降雨误差估计}

对于一场洪水, 假如有 $m$ 个时段雨量, 其相应的雨量误 差依时间顺序为 $e_{P_{1}}, e_{P_{2}}, \cdots, e_{P_{m}}$, 假如有 $L$ 个时段的流量系 列, 流量的误差序列为 $e_{Q_{1}}, e_{Q_{2}}, \cdots, e_{Q_{L}}$, 则降雨误差与流量误 差之间的关系可以表达为式 (10) :

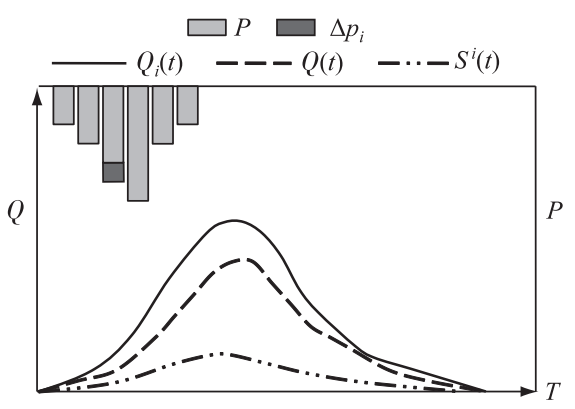

图 2 降雨系统响应曲线示意图 Fig.2 The schematic diagram of rainfall system response curve 


$$
\left\{\begin{array}{c}
e_{Q_{1}}=S_{11} \cdot e_{P_{1}}+S_{12} \cdot e_{P_{2}}+\cdots+S_{1 m} \cdot e_{P_{m}} \\
e_{Q_{2}}=S_{21} \cdot e_{P_{1}}+S_{22} \cdot e_{P_{2}}+\cdots+S_{2 m} \cdot e_{P_{m}} \\
\vdots \\
e_{Q_{L}}=S_{L 1} \cdot e_{P_{1}}+S_{L 2} \cdot e_{P_{2}}+\cdots+S_{L m} \cdot e_{P_{m}}
\end{array}\right.
$$

式(10) 表达为向量矩阵形式为:

$$
E_{Q}=S \cdot E_{P}
$$

式中, $E_{Q}=\left[\begin{array}{c}e_{Q_{1}} \\ \vdots \\ e_{Q_{L}}\end{array}\right], S=\left[\begin{array}{ccc}S_{11} & \cdots & S_{1 m} \\ & \vdots & \\ S_{L 1} & \cdots & S_{L m}\end{array}\right], E_{P}=\left[\begin{array}{c}e_{P_{1}} \\ \vdots \\ e_{P_{m}}\end{array}\right]$, 式 (11) 表达了降雨误差与流量计算误差的响应关系.

则面平均雨量误差与流量误差之间的系统响应关系为:

面平均雨量误差的最小二乘估计值为:

$$
E_{Q}=S \cdot E_{P}+\zeta
$$

$$
E_{P}^{\prime}=\left(S^{T} \cdot S\right)^{-1} \cdot S^{T} \cdot E_{Q}
$$

式中, $\zeta$ 为残差向量, $E_{P}^{\prime}$ 为降雨误差的动态系统响应曲线估计向量. 由式 (13)计算得到的面平均雨量误差系 列加上原来的面平均雨量系列重新使用预报模型计算, 便可得到通过降雨系统响应曲线反馈修正以后的流 域出口断面流量过程.

\section{2 应用检验}

在本研究中将基于面平均雨量误差修正的实时洪水预报修正方法与新安江模型相结合, 对此修正方法 进行理想案例和实际流域应用检验. 本研究之所以选取新安江模型, 是因为新安江模型是一个概念性集总 式水文模型, 其模型输人项为流域蒸散发能力和单元面平均雨量, 而本文所提出的修正方法恰恰是通过修 正面平均雨量来提高洪水预报精度, 且本文所选取的应用流域为淮河上游王家坝流域, 此流域为半湿润流 域,符合新安江模型的应用范围.

理想模型和实际流域检验应用采用以下评价指标:

(1) NS( Nash-Sutcliffe) 系数:

$$
N S=1-\sum_{j=1}^{L}\left(M_{\mathrm{C}}(j)-M_{0}(j)\right)^{2} / \sum_{j=1}^{L}\left(M_{0}(j)-\overline{M_{0}}\right)^{2}
$$

(2) 相对误差:

$$
\Delta M(\%)=\left[\left(M_{\mathrm{C}}-M_{0}\right) / M_{0}\right] \times 100 \%
$$

(3) 修正后相对误差提高幅度:

$$
I R M=\left|R M_{\mathrm{bu}}-R M_{\mathrm{au}}\right| /\left|R M_{\mathrm{bu}}\right| \times 100 \%
$$

(4) NS 系数提高幅度:

$$
I N S=\left(N S_{\mathrm{bu}}-N S_{\text {au }}\right) /\left(1-N S_{\mathrm{bu}}\right) \times 100 \%
$$

式中, $M_{0}$ 为统计量的实测值, $M_{\mathrm{C}}$ 为统计量的计算值, $\overline{M_{0}}$ 为实测系列的平均值. $R M_{\mathrm{bu}}$ 表示某一统计指标修正 前的值, $R M_{\mathrm{au}}$ 表示统计指标修正之后的值, $I R M$ 表示统计指标在修正前后提高的百分比 $(R M$ 可以是径流深 相对误差或者洪峰相对误差), 其值越高表示提高的幅度越大, 修正的效果更加明显. $N S_{\mathrm{bu}}$ 表示修正前的 $N S$ 系数, $N S_{\text {au }}$ 表示修正后的 $N S$ 系数.

\section{1 理想案例应用检验}

理想案例中使用的流域面积为 $2000 \mathrm{~km}^{2}$, 流域中雨量站个数为 5 , 马斯京根河道演算的总河段数也为 5 . 以雨量站为依据, 将流域按照平均权重划分为 5 个流域子单元. 本理想案例中 5 个子单元的面平均雨量以 及雨量误差均给定, 使用本文提出的修正方法估计面平均雨量误差, 然后将估计得到的雨量误差与给定的 误差系列进行对比, 以此来验证此方法理论依据是否可行. 在此理想案例中, 5 个单元的面平均雨量资料以 及每个站的时段雨量的误差均不同. 详细的降雨资料、降雨误差和降雨误差的估计值见表 1 . 
表 1 中 $P_{1} \sim P_{5}$ 分别表示 5 个雨量站单元的面平均 雨量, 为了保证给定的雨量数据更加接近一场洪水的 降雨过程, 其具体降雨数据采用湖北省乌溪沟流域 2012 年 8 月 15 日一场历史洪水降雨资料. 采用流域 内 8 个雨量站中的 5 个雨量站数据来作为本理想案例 中 5 个雨量站单元的面平均雨量. $\Delta P$ 表示给定的雨 量误差, 误差值为服从零均值分布的随机误差, 误差的 控制范围为时段面平均雨量值的 $\pm 20 \%$ 以内. $\Delta P^{\prime}$ 表示 使用降雨系统响应曲线方法估计的面平均雨量误差 值. 在本理想案例中, 由于流域出口断面的实测流量 系列只有 1 个,因此本研究中估计出的面平均雨量误 差为整个流域的时段面平均雨量误差值,而非每个雨 量单元的面平均雨量误差值. 因此, 本理想案例检验 中只给定全流域的面平均雨量误差系列.

给定的面平均雨量误差系列与通过降雨系统响应 曲线方法估计得到的面平均雨量误差系列对比图如图 3 所示.

理想案例修正前、后流量过程线对比结果如图 4 所示. 图中 $P$ 表示降雨, $Q_{\mathrm{c}}$ 表示流量计算系列, $Q_{\mathrm{u}}$ 表示 修正后的计算流量, $Q_{0}$ 表示实测流量过程. 理想案例 中没有实测流量,本理想案例中的实测流量系列是由 下式计算得到, 即：

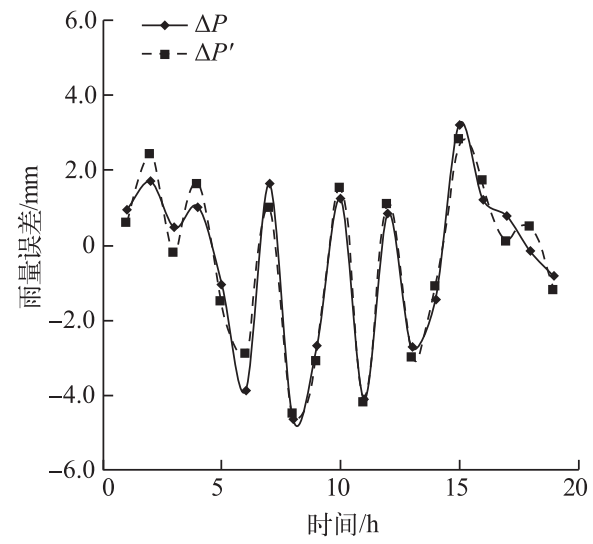

图 3 给定雨量误差与估计雨量误差对比

Fig.3 Comparison between the given rainfall errors and the estimated rainfall errors
表 1 理想案例中雨量、雨量误差和误差估计值 Tab.1 The rainfall data, the given rainfall errors and estimated rainfall errors in ideal case

\begin{tabular}{rrrrrrrr}
\hline$T /$ & $P_{1} /$ & $P_{2} /$ & $P_{3} /$ & $P_{4} /$ & $P_{5} /$ & $\Delta P /$ & $\Delta P^{\prime} /$ \\
$\mathrm{h}$ & $\mathrm{mm}$ & $\mathrm{mm}$ & $\mathrm{mm}$ & $\mathrm{mm}$ & $\mathrm{mm}$ & $\mathrm{mm}$ & $\mathrm{mm}$ \\
\hline 1 & 5.1 & 5.1 & 5.4 & 3.1 & 2.0 & 1.0 & 0.6 \\
2 & 2.1 & 2.9 & 4.9 & 3.3 & 3.9 & 1.7 & 2.4 \\
3 & 4.6 & 4.9 & 3.7 & 2.7 & 2.9 & 0.5 & -0.2 \\
4 & 10.5 & 12.1 & 5.6 & 10.4 & 9.3 & 1.0 & 1.6 \\
5 & 8.4 & 5.9 & 6.0 & 6.6 & 9.9 & -1.1 & -1.5 \\
6 & 7.3 & 8.9 & 11.0 & 13.7 & 12.1 & -3.9 & -2.9 \\
7 & 15.0 & 15.5 & 7.8 & 15.9 & 12.6 & 1.6 & 1.0 \\
8 & 8.8 & 7.2 & 9.6 & 12.7 & 6.9 & -4.6 & -4.5 \\
9 & 12.9 & 18.3 & 14.1 & 10.6 & 15.3 & -2.7 & -3.1 \\
10 & 17.9 & 15.6 & 14.1 & 17.3 & 10.3 & 1.2 & 1.5 \\
11 & 12.6 & 8.5 & 9.1 & 14.5 & 20.7 & -4.1 & -4.2 \\
12 & 16.5 & 13.9 & 12.2 & 11.1 & 18.1 & 0.9 & 1.1 \\
13 & 11.8 & 12.6 & 17.6 & 13.8 & 14.0 & -2.7 & -3.0 \\
14 & 10.8 & 5.6 & 15.0 & 13.8 & 16.2 & -1.4 & -1.1 \\
15 & 13.0 & 10.2 & 7.8 & 8.1 & 11.3 & 3.2 & 2.8 \\
16 & 11.0 & 13.1 & 9.2 & 8.1 & 9.7 & 1.2 & 1.7 \\
17 & 10.6 & 10.7 & 11.3 & 5.9 & 12.1 & 0.8 & 0.1 \\
18 & 6.3 & 7.5 & 4.8 & 3.9 & 3.3 & -0.2 & 0.5 \\
19 & 2.3 & 1.5 & 3.6 & 1.7 & 2.8 & -0.8 & -1.2 \\
\hline & & & & & & &
\end{tabular}

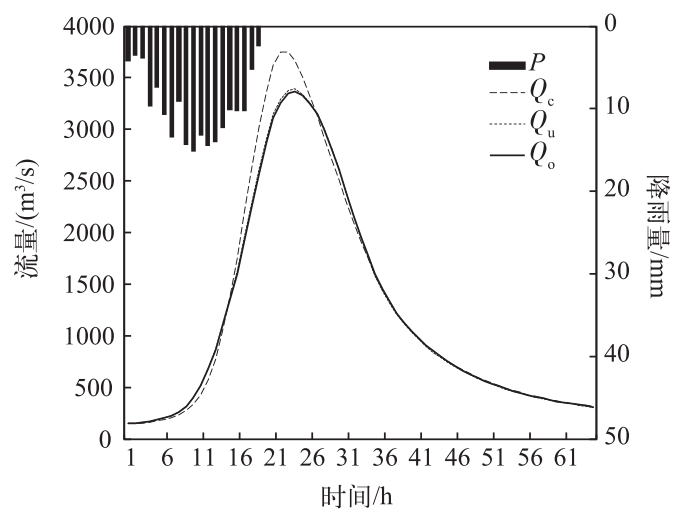

图 4 理想案例流量过程对比

Fig.4 The hydrographs comparison of ideal case

$$
Q_{\mathrm{o}}=Q(P+\Delta P)+\varepsilon
$$

式中, $Q(P+\Delta P)$ 表示使用面平均雨量系列 $P+\Delta P$ 计算得到的流量系列, $\varepsilon$ 是零均值的随机流量误差, 误差范 围控制在对应时段流量的 \pm \% .

通过图 3 和图 4 可以看出,在各站之间降雨资料以及每个站的时段雨量的误差均不同的情况下,使用降 雨系统响应曲线方法估计的面平均雨量误差和给定的误差系列非常接近, 2 个系列的 NS 系数高达 0.946 . 给 定面平均雨量误差的总和为 $-8.4 \mathrm{~mm}$, 通过动态系统响应曲线方法估计的降雨误差总和为 $-8.4 \mathrm{~mm}$, 相对误 
差为 0 , 满足水量平衡. 洪峰相对误差由修正前的 $11.3 \%$ 减小到 $0.7 \%$, 径流深相对误差由修正前的 $3.48 \%$ 降 低到 $0.24 \%$, 洪水流量过程确定性系数由修正前的 0.834 提高到了 0.992 . 在实测流量存在随机误差的前提 下, 通过此方法能够准确地估计出面平均雨量误差系列, 且能够有效地修正流域出口断面流量过程, 提高预 报精度,说明此方法理论可行,能够有效地提高预报效果, 可以将此方法应用于实际流域检验.

\section{2 实际流域应用}

对于空间集总式水文模型, 流域的大小以及流域内雨量站密度会对面平均雨量计算有较大影响. 鉴于 此, 本研究不仅将此方法应用于王家坝流域进行检验, 而且对王家坝流域不同雨量站密度情况的应用效果 进行研究, 分析不同雨量站密度对此方法修正效果的影响.

王家坝流域面积为 $30500 \mathrm{~km}^{2}$, 王家坝水文站是淮河干流上的重要控制站,此流域对于整个流域的洪水 控制和管理起着重要作用. 王家坝水文站以上 $90 \%$ 的研究区域位于河南境内的东南部, 流域主要由丘陵、山 区和平原组成, 流域内共有 41 个雨量站. 研究区域的气候为亚热带湿润季风气候, 多年平均降雨量为 940 $m m(1953-2000 \text { 系列 })^{[21]}$. 本研究中将王家坝流域雨量站点分布情况分为 3 种, 站点水系图如图 5 所示.

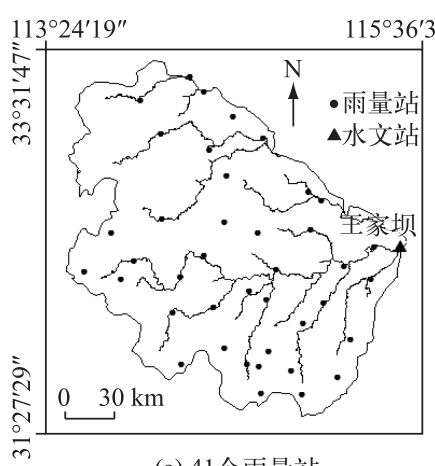

(a) 41 个雨量站

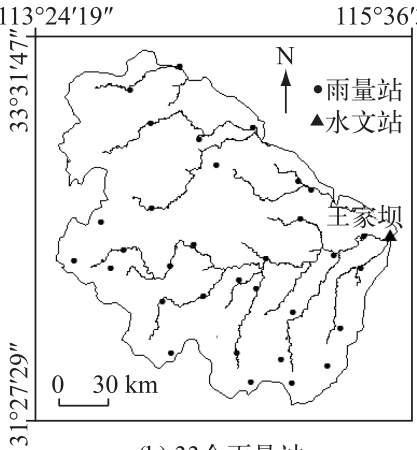

(b) 33 个雨量站

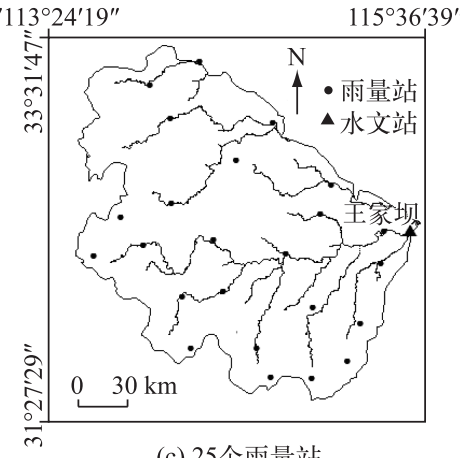

(c) 25 个雨量站

图 5 王家坝流域 3 种不同站点密度的水系

Fig.5 Three different rain gauge distributions of Wangjiaba Basin

图 5 中 b 图雨量站分布情况为将原有 41 个雨量站随机去掉 8 个, $\mathrm{c}$ 图为在 b 图的基础上再随机去掉 8 个. 本文选取了王家坝流域 1984- 2009 年共 16 场历史洪水进行验证. 修正过程中所使用的模型参数是经

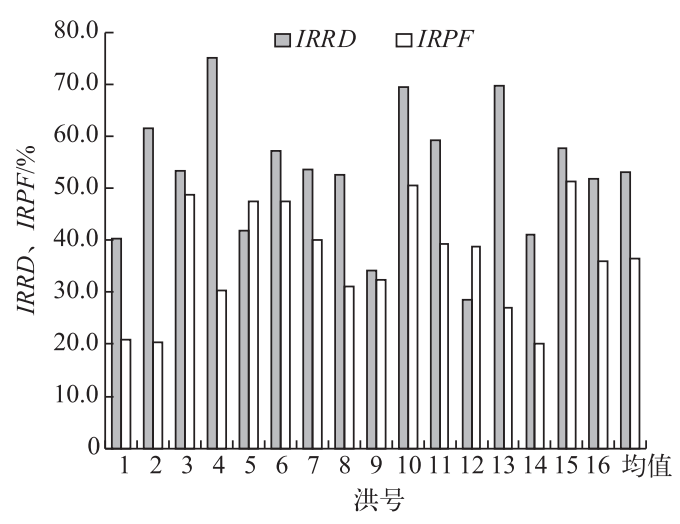

图 6 王家坝流域 16 场洪水的 $I R R D$ 和 IRPF 效果对比

Fig.6 The comparison between IRRD and $I R P F$ of 16 flood events in Wangjiaba Basin 过王家坝流域 16 场历史洪水率定检验后获得. 16 场历史洪水修正前后计算结果, 以及不同雨量站分 布情况修正效果提高的幅度统计结果见表 2.

在雨量站个数不减少的情况下, 16 场历史洪水 的预报精度均有明显提高 (表 2). 平均 $N S$ 系数由修 正前的 0.748 提高到 0.905 , 提高了 0.157 . 径流深相 对误差绝对值的平均值由修正前的 $9.6 \%$ 减小到 $4.5 \%$, 减小了 $5.1 \%$. 洪峰相对误差绝对值的平均值 由修正前的 $13.4 \%$ 减小到 $8.5 \%$, 减小了 $4.9 \%$. 结果 显示此修正方法对于洪水径流深和洪峰误差均有明 显的修正效果. 对于径流深误差的修正幅度明显大 于对于洪峰误差的修正幅度 (图 6), 说明此方法对 于控制洪水预报过程中水量平衡具有明显效果.

在不同的雨量站密度条件下, 此方法对于径流 深、洪峰流量和 NS 系数修正效果的对比如图 7 所示. 
表 2 不同雨量站分布情况下修正结果对比 *

Tab.2 The calculated results of different rainfall gauge distributions

\begin{tabular}{|c|c|c|c|c|c|c|c|c|c|c|c|c|c|c|c|}
\hline \multirow{2}{*}{ 洪号 } & \multicolumn{5}{|c|}{41 个雨量站 } & \multicolumn{5}{|c|}{33 个雨量站 } & \multicolumn{5}{|c|}{25 个雨量站 } \\
\hline & $N S_{\text {bu }}$ & $N S_{\text {au }}$ & IRRD & $I R P F$ & INS & $N S_{\text {bu }}$ & $N S_{\text {au }}$ & $I R R D$ & IRPF & INS & $N S_{\text {bu }}$ & $N S_{\text {au }}$ & $\operatorname{IRRD}$ & $I R P F$ & INS \\
\hline 1 & 0.855 & 0.930 & 40.4 & 20.9 & 51.7 & 0.823 & 0.924 & 42.1 & 32.0 & 57.1 & 0.784 & 0.914 & 70.5 & 34.1 & 60.2 \\
\hline 2 & 0.793 & 0.939 & 61.5 & 20.3 & 70.5 & 0.761 & 0.931 & 62.5 & 29.5 & 71.1 & 0.701 & 0.911 & 66.7 & 30.2 & 70.2 \\
\hline 3 & 0.731 & 0.922 & 53.4 & 48.8 & 71.0 & 0.700 & 0.915 & 54.5 & 49.6 & 71.7 & 0.679 & 0.904 & 54.7 & 50.3 & 70.1 \\
\hline 4 & 0.761 & 0.896 & 75.0 & 30.4 & 56.5 & 0.704 & 0.878 & 71.8 & 33.9 & 58.8 & 0.671 & 0.869 & 65.2 & 33.5 & 60.2 \\
\hline 5 & 0.685 & 0.867 & 41.9 & 47.4 & 57.8 & 0.647 & 0.854 & 43.4 & 48.4 & 58.6 & 0.625 & 0.848 & 43.7 & 47.3 & 59.5 \\
\hline 6 & 0.717 & 0.919 & 57.1 & 47.5 & 71.4 & 0.693 & 0.913 & 58.5 & 53.1 & 71.7 & 0.643 & 0.904 & 64.4 & 53.3 & 73.1 \\
\hline 7 & 0.743 & 0.879 & 53.5 & 40.0 & 52.9 & 0.723 & 0.874 & 54.5 & 42.6 & 54.5 & 0.701 & 0.865 & 55.0 & 47.0 & 54.8 \\
\hline 8 & 0.721 & 0.918 & 52.6 & 31.2 & 70.6 & 0.701 & 0.913 & 53.4 & 35.5 & 70.9 & 0.689 & 0.907 & 54.4 & 44.9 & 70.1 \\
\hline 9 & 0.764 & 0.845 & 34.3 & 32.4 & 34.3 & 0.714 & 0.838 & 36.7 & 41.3 & 43.4 & 0.699 & 0.830 & 38.4 & 50.6 & 43.5 \\
\hline 10 & 0.732 & 0.925 & 69.4 & 50.4 & 72.0 & 0.712 & 0.920 & 71.7 & 55.6 & 72.2 & 0.693 & 0.911 & 67.5 & 55.2 & 71.0 \\
\hline 11 & 0.721 & 0.914 & 59.2 & 39.3 & 69.2 & 0.703 & 0.909 & 62.8 & 39.0 & 69.4 & 0.675 & 0.902 & 64.0 & 43.3 & 69.8 \\
\hline 12 & 0.707 & 0.927 & 28.6 & 38.9 & 75.1 & 0.701 & 0.920 & 34.7 & 40.0 & 73.2 & 0.668 & 0.911 & 39.2 & 42.4 & 73.2 \\
\hline 13 & 0.715 & 0.874 & 69.7 & 27.1 & 55.8 & 0.699 & 0.868 & 71.3 & 30.8 & 56.1 & 0.645 & 0.861 & 64.6 & 28.9 & 60.8 \\
\hline 14 & 0.722 & 0.908 & 41.2 & 20.1 & 66.9 & 0.701 & 0.901 & 43.5 & 20.8 & 66.9 & 0.674 & 0.893 & 44.5 & 25.7 & 67.2 \\
\hline 15 & 0.870 & 0.922 & 57.8 & 51.2 & 40.0 & 0.825 & 0.911 & 60.3 & 50.5 & 49.1 & 0.775 & 0.905 & 61.1 & 51.5 & 57.8 \\
\hline 16 & 0.733 & 0.902 & 51.7 & 36.1 & 63.3 & 0.713 & 0.897 & 55.6 & 35.8 & 64.1 & 0.677 & 0.882 & 48.3 & 36.5 & 63.5 \\
\hline 均值 & 0.748 & 0.905 & 53.0 & 36.4 & 61.2 & 0.720 & 0.898 & 54.8 & 39.9 & 63.1 & 0.687 & 0.889 & 56.4 & 42.2 & 64.1 \\
\hline
\end{tabular}

* 在本研究中用 $I R R D$ 表示径流深相对误差提高幅度,IRPF 表示洪峰相对误差提高幅度,IRRD、IRPF 和 INS 在表格中的 具体数据均为百分数 $(\%)$.

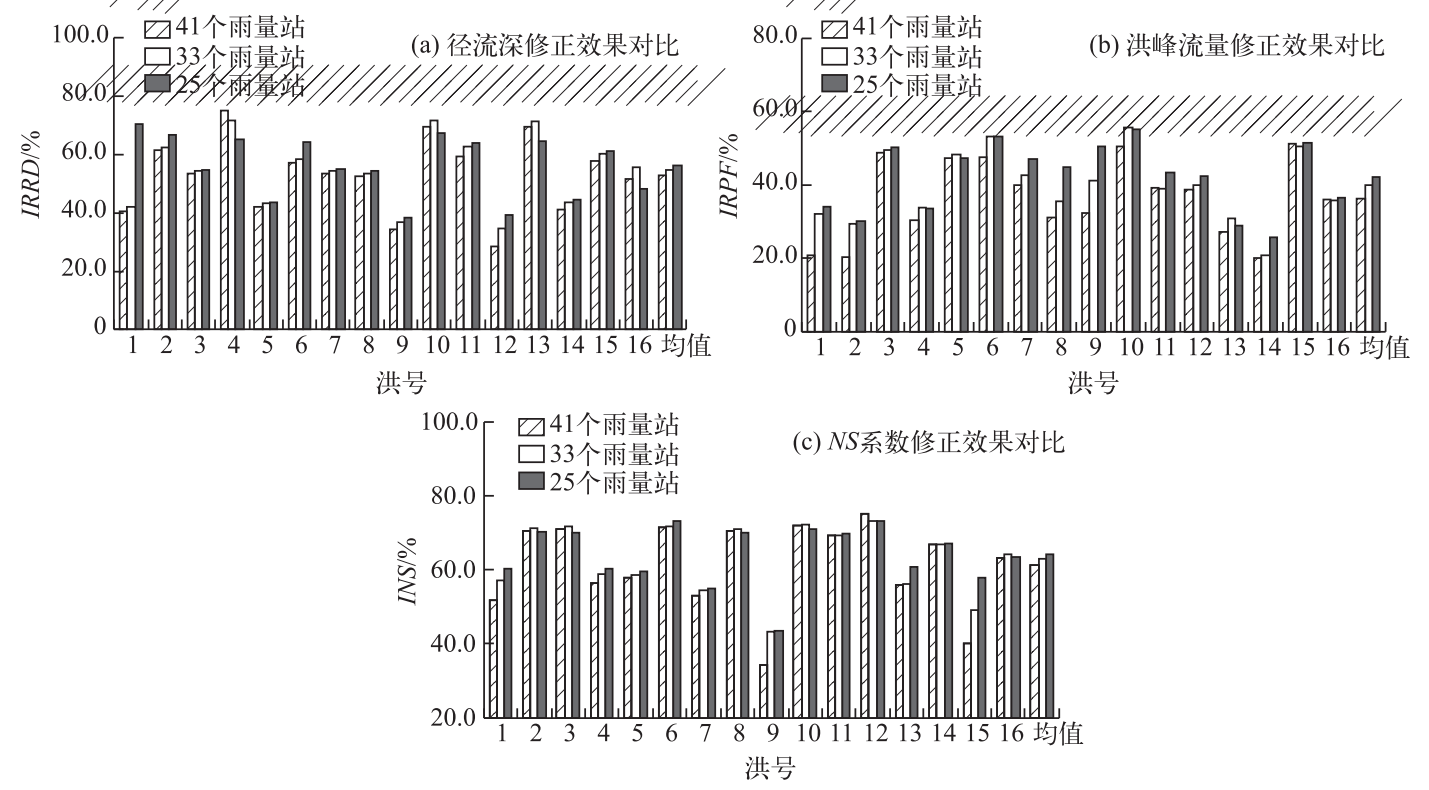

图 7 王家坝流域不同雨量站密度下修正效果提高幅度对比

Fig.7 The improvement comparisons of different rain gauge densities in Wangjiaba Basin

降雨系统响应曲线修正方法在 3 种不同雨量站密度情况下对于径流深和洪峰流量的平均修正效果以 及平均 $N S$ 系数均有明显提高. 雨量站密度越低, 其修正效果提高的幅度越大 (图 7). 雨量站密度低, 造成模 


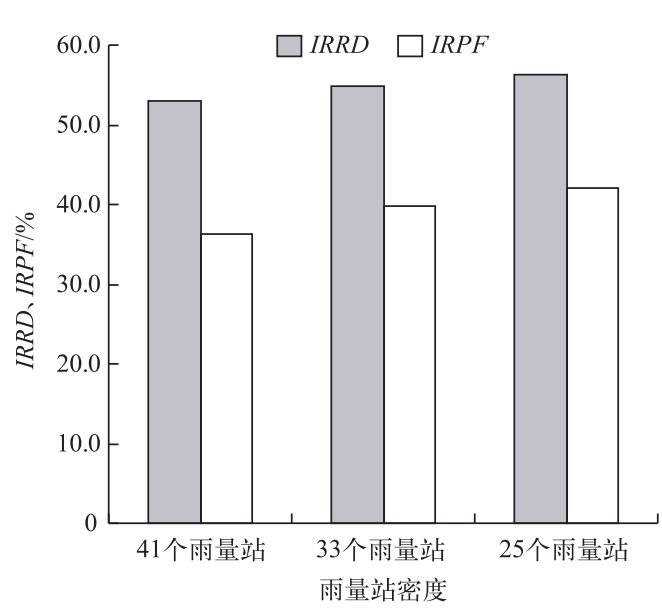

图 8 王家坝流域不同雨量站密度 下 $I R R D$ 与 $I R P F$ 对比结果

Fig. 8 The comparison of IRRD and IRPF of different rain gauge densities in Wangjiaba Basin
型输人项不确定性增加, 面平均雨量误差较大, 通过降 雨系统响应曲线方法能够有效降低面平均雨量的不确 定, 从而提高模型预报精度. 因此, 在没有误差修正时 采用预报模型进行洪水预报计算, 洪水预报精度相对 偏低, 修正的效果就比较明显. 随着雨量站个数的增 加, 雨量站密度增大, 面平均雨量精度有所提高, 预报 精度相对增加, 在此情况下修正效果的提高幅度就有 所减小.

3 种雨量站密度情况下, 本修正方法对于径流深 修正效果的平均提高幅度均比洪峰流量修正效果的提 高幅度大, 进一步说明此方法能够更好的修正洪水预 报过程中水量平衡误差 (图 8).

通过此方法在王家坝流域的应用结果以及在不同 雨量站密度情况下的结果分析, 表明此方法能够有效 提高实际流域洪水预报的精度, 且对于雨量站点密度 较低的流域修正效果更加显著.

\section{3 结论}

本文提出了一种基于降雨系统响应曲线的面平均雨量修正方法, 通过估计面平均雨量误差来提高水文 模型的预报精度. 将水文模型概化为系统, 以响应函数对面平均降雨系列的偏微分为降雨系统响应曲线的 理论基础. 建立了面平均雨量误差与流域出口断面流量误差之间的系统响应关系. 利用实测流量与计算流 量之间的差值系列, 通过最小二乘估计面平均雨量的误差系列, 使用修正之后的面平均雨量系列重新计算 流域出口端面的出流过程, 以此提高模型预报精度. 虽然此方法对于提高洪水预报精度具有一定的效果, 但 由于水文系统毕竟是非线性系统, 影响因素较为复杂, 这两者之间不是绝对的线性关系, 且预报流量过程的 误差并不是全部由面平均雨量误差所引起的. 因此, 如何细化面平均雨量误差和计算流量误差之间的响应 机制和量化关系, 以及面平均雨量误差和其他误差因素的量化影响还有待进一步研究.

现阶段的研究结果表明,通过修正面平均雨量误差来提高洪水预报精度是一种有效的预报误差修正手 段, 本修正方法通过理想案例和实际流域验证, 理想案例中次洪径流深相对误差由修正前的 $3.48 \%$ 减小到 $0.24 \%$, 洪水流量过程确定性系数由修正前的 0.834 提高到了 0.992 ; 实际流域应用结果为径流深相对误差绝 对值的平均值由修正前的 $9.6 \%$ 减小到 $4.5 \%$, 平均 $N S$ 系数由修正前的 0.748 提高到 0.905 , 结果证明此降雨 系统响应曲线方法能够有效地修正面平均雨量误差, 提高洪水预报精度. 此外, 还将此方法应用于王家坝流 域的不同雨量站密度情况下, 应用结果显示对于不同的雨量站分布情况均有明显的修正效果. 对于雨量站 密度较低的流域, 此方法降低面平均雨量的不确定性效果更加显著, 洪水预报精度提高幅度更加明显, 对此 方法的应用范围和适用条件具有一定的指导意义.

本研究提出的修正方法具有结构简单、不增加模型参数、不改变复杂度以及不增加模型中间变量的优 点, 是一种有效的提高洪水预报精度的修正方法, 此修正方法有待更广泛的应用和检验. 此外, 本方法现阶 段是利用具有较好条件的水文测站流域进行研究, 水库流域的人库流量过程具有一定的特殊性, 由于水位 波动造成反推的人库流量过程呈现锯齿状, 从实测流量过程中提取面平均雨量误差信息具有一定的难度, 如何通过处理人库流量过程将此方法能够应用于水库流域的面平均雨量误差修正值得进一步的探索研究. 本文通过分析研究面平均雨量来修正洪水预报误差, 面平均雨量的估计也可以通过降雨等值线图来动态计 算得到, 在后续的研究中可尝试将雨量等值线图方法与本文中提出的方法进行探讨和对比研究, 对于研究 面平均雨量误差与洪水预报精度之间的量化关系具有一定的参考价值.

\section{4 参考文献}

[ 1 ] Komma J, Bloschl G, Reszler C. Soil moisture updating by Ensemble Kalman Filtering in real-time flood forecasting. Jour- 
nal of Hydrology, 2008, 357(3/4) : 228-242.

[ 2 ] Lee WL, Eugene LP. Accuracy of precipitation measurements for hydrologic modeling. Water Resources Research, 1974,10 (4) : 857-863.

[ 3 ] Hoshin G, Martyn PC, JASPER AV et al. Towards a comprehensive assessment of model structural adequacy. Water Resources Research, 2012, 48, W08301. DOI: 10.1029/2011WR011044.

[ 4 ] Jasper AV, Hoshin G, Luis AB et al. Effective and efficient algorithm for multi-objective optimization of hydrologic models. Water Resources Research, 2003, 39(8) : 1214. DOI:10.1029/2002WR001746.

[ 5 ] Fan C, Wade TC, Patrick JS et al. Improving hydrologic predictions of a catchment model via assimilation of surface soil moisture. Advances in Water Resources, 2011, 34: 526-536.

[ 6 ] Bogner K, Kalas M. Error-correction methods and evaluation of an ensemble based hydrological forecasting system for the Upper Danube catchment. Atmospheric Science Letters, 2008, 9(2) : 95-102.

[ 7 ] Marco F, Anna B, Silvia B et al. Forecasting discharges at the downstream end of a river reach through two simple Muskingum based procedures. Journal of Hydrology, 2011, 399: 335-352.

[ 8 ] Jeffrey CN, Peter MA, Craig WH. Evaluating the utility of the ensemble transform Kalman filter for adaptive sampling when updating a hydrodynamic model. Journal of Hydrology, 2009, 375: 589-600.

[ 9 ] Moradkhani H, Dechant CM, Sorooshin S. Evolution of ensemble data assimilation for uncertainty quantification using the particle Filter-Markov Chain Monte Carlo method. Water Resources Research, 2012, 48 ( 12): W12520. DOI: 10. 1029/2012WR012144.

[10] Weerts AH, El SG. Particle filtering and ensemble Kalman filtering for state updating with hydrological conceptual rainfallrunoff models. Water Resources Research, 2006, 42(9): W09403. DOI: 10.1029/2005WR004093.

[11] Qu SM, Bao WM. Comprehensive correction of real-time flood forecasting. Advances in Water Science, 2003, 14(2) : 167171. [翟思敏, 包为民. 实时洪水预报综合修正方法初探. 水科学进展, 2003, 14(2): 167-171.]

[12] Bao WM, Si W, Shen GH et al. Runoff error updating based on unit hydrograph inversion. Journal of Advances in Water Science, 2012, 23(3) : 315-322. [包为民, 司伟, 沈国华等. 基于单位线反演的产流误差修正. 水科学进展, 2012, $\mathbf{2 3}(3)$ : 315-322.]

[13] Si Wei, Bao Weimin, Qu Simin. Runoff error correction in real-time flood forecasting based on dynamic system response curve. Journal of Advances in Water Science, 2013, 24(4)：497-503. [司伟, 包为民, 翟思敏. 洪水预报产流误差的 动态系统响应曲线修正方法. 水科学进展, 2013, 24(4): 497-503.]

[14] Bao W, Si W, Qu S. Flow updating in real-time flood forecasting based on runoff correction by dynamic system response curve. Journal of Hydrologic Engineering, 2014, 19: 747-756.

[15] Sionghuat C, Rafael LB. Optimal estimators of mean areal precipitation in regions of orographic influence. Journal of Hydrology, 1982, $57: 23-48$.

[16] Golder J, Joelson M, Neel MC et al. A time fractional model to represent rainfall process. Water Science and Engineering, 2014, 7(1) : 32-34.

[17] Krajewski WF, Smith JA. Radar hydrology : rainfall estimation. Advances in Water Resources, 2002, 25: 1387-1394.

[18] Moriasi DN, Arnold JG, Liew MWV et al. Model evaluation guidelines for systematic quantification of accuracy in watershed simulations. Transactions of the Asabe, 2007, 50(3): 885-900.

[19] Madsen H. Automatic calibration of a conceptual rainfall-runoff model using multiple objectives. Journal of Hydrology, 2000, 235: 276-288.

[20] Hamid M, Soroosh S, Hoshin G et al. Dual state-parameter estimation of hydrological models using ensemble Kalman filter. Advances in Water Resources, 2005, 28: 135-147.

[21] Li FG. Study on flood forecasting and regulating of the Linhuaigang project on Huaihe river [Dissertation]. Nanjing: Hohai University, 2007: 13-25. [李富贵. 淮河临淮岗工程洪水预报调度研究 [学位论文]. 南京: 河海大学, 2007: 13-25.] 